\title{
Effects of a novel dye containing toothpaste versus regular fluoridated toothpaste for supragingival biofilm reduction in patients with chronic gingivitis: A Randomized Controlled
} Trial

\author{
Avita Rath, Chong Jun Ning, Priyadarshini HR, Brandon Nicholas Teo, Naik Hsian Zhen, Ong Wey Yin
}

SEGi University, Malaysia

\section{Abstract}

\begin{abstract}
AIM: To compare the effects of a plaque disclosing toothpaste with a regular fluoridated toothpaste for supragingival biofilm reduction in patients with chronic gingivitis.
\end{abstract}

MATERIALS AND METHODS: This study was a randomized controlled trial with a parallel design. Forty patients, both males and females aged 18-25 years with chronic generalized gingivitis ( $N=40)$ were randomly allocated to two groups of twenty each $(n=20)$, using lottery method. Participants in the experimental group brushed with a plaque disclosing toothpaste, while participants in the control group brushed with regular fluoridated toothpaste. Clinically, biofilm was measured using Silness \& Loe Plaque Index, both at baseline and at follow up visits by two assessors who were blinded to group allocation. Adjunctly, intraoral photographs were taken for Digital Plaque Image Analysis of the remaining dental biofilm in the upper and lower anterior labial tooth surfaces, and calculated based on the area of stained pixels. Data was analyzed using SPSS version 22 . Mean plaque scores between the groups were compared using independent sample t-test and $p<0.05$ was considered significant.

RESULTS: Data of all the 40 participants were analyzed as they were no drop outs. No significant differences were found in the mean plaque scores between the two groups $(p=0.059)$ at the end of the study. Similarly, Digital Plaque Image Analysis showed no significant difference in the mean plaque scores between the groups $(p=0.93)$.
CONCLUSION: This study showed that there are no additional benefits of using a plaque disclosing toothpaste, in enhancing the removal of the supragingival dental biofilm during routine oral hygiene maintenance.

KEYWORDS: dental biofilm; supragingival; gingivitis; oral hygiene; dentifrice

Citation: Rath, A et al. (2021) Effects of a novel dye containing toothpaste versus regular fluoridated toothpaste for supragingival biofilm reduction in patients with chronic gingivitis: A Randomized Controlled Trial

Dentistry 3000. 1:a001 doi:10.5195/d3000.2021.117

Received: September 10, 2020

Accepted: January 16, 2021

Published: June 21, 2021

Copyright: (02021 Rath, A et al. This is an open access article licensed under a Creative Commons Attribution Work 4.0 United States License.

Email: reachdocpriya@gmail.com

\section{Introduction}

Dental biofilm is the primary cause for many oral diseases and conditions including periodontitis [1]. Currently, periodontal disease is estimated to be the 11th most prevalent disease globally [2]. Periodontitis can result in tooth loss and worsening of certain systemic illness, leading to a compromised quality of life [2]. Thus, adequate plaque control should be the primary goal of daily oral hygiene, as it prevents the occurrence of oral diseases and future tooth loss $[3,4]$.
Even though tooth brushing is the cornerstone of home oral hygiene practices, the high incidence and prevalence of periodontal problems globally reveal that these home hygiene practices are not adequate to maintain good oral hygiene [4-6]. 
While brushing is the most widespread and universally suitable measure for the mechanical removal of the dental biofilm, it should be associated with constant motivation [7-9].

Incomplete biofilm removal may be partly attributed to the invisible nature of dental biofilm. This can be overcome by adding a dye, thereby disclosing the biofilm, especially in the posterior retentive areas. Increasing the visibility of the pathological source to patients can be a promising and reliable way to improvise their home oral hygiene practices, and thus can motivate them to achieve ideal brushing technique [10]. Studies suggest that the use of disclosing agents such as erythrosine, will help to remove dental biofilm more easily when used prior to brushing [11]. However, there is still a wide gap in knowledge regarding the effectiveness of such products in home-use.

Various indices have been used to quantify dental biofilm in different clinical and research settings. Though these indices quantify biofilm in an objective manner, they are not devoid of certain limitations such as subjective assessment of the operator [12-14]. Several authors have proposed other methods such as the use of grids and planimetric systems including the use of fluorescent light to visualize and measure biofilm $[12,15]$.
The assessment of the outcome of oral hygiene practices needs tools with higher accuracy, consistency and sensitivity towards the amount of remaining biofilm on the surface of the teeth [16]. Digital plaque image analysis (DPIA) could be a noninvasive alternative for measuring biofilm on the tooth surface and has been used in some studies [10]. However, majority of the studies so far have evaluated the efficacy of dye containing toothpaste in professional care, hence it warranted its evaluation in self-care as well. Hence, the main objective of the present study was to compare the effectiveness of a plaque-disclosing toothpaste and a regular fluoridated toothpaste in removing the supragingival biofilm, using DPIA as an adjunct to clinical measurement, during routine oral hygiene maintenance.

\section{Material and Methods}

This was a randomized controlled trial with two parallel arms. The trial was registered at ClinicalTrials.gov with the ID No NCT03287011 prospectively.

\section{Ethical considerations}

The study was approved by the Institutional Ethics Committee with EC number SEGi/EC/2017-18/11. Informed and written consent was obtained from all the participants prior to the commencement of the trial. The trial followed the CONSORT guidelines and was designed in compliance with the Declarations of Helsinki Act [17], with principles of Good Clinical Practice.

\section{Study participants}

Study participants included healthy male and female patients in the age range of $18-25$ years visiting SEGi oral health centre, Kota Damansara, Malaysia, with chronic generalized gingivitis. Patients who were pregnant or lactating, who had used anti-inflammatory and antibiotics two weeks prior, undergone any dental treatment within the past 30 days, and persons undergoing orthodontic treatment were excluded from the study. One hundred and seven people were screened for eligibility between January 2018 and March 2018 and forty of them were recruited for the study based on the inclusion and the exclusion criteria (Fig.1).

\section{Sample size}

The sample size was calculated using G power software. With a $95 \%$ confidence interval, $80 \%$ power and an effect size of $90 \%$, a sample size of $\mathrm{n}=40$ (20 in each group) was determined.

\section{Randomization and Concealment}

Study participants were randomized into two groups based on the lottery method. Allocation concealment was ensured by providing sealed envelopes with participants' 


\section{Control Group A $(\mathbf{n}=\mathbf{2 0})$}

- $\quad$ Review Informed Consent

- Confirm appropriate hygiene

- Brush teeth with control toothpaste

- Take intraoral photo

- $\quad$ Silness \& Loe Plaque Index

- Dismiss participants

- Confirm appropriate hygiene cessation

- Brush teeth with control toothpaste

- Take intraoral photo

- $\quad$ Silness \& Loe Plaque Index

- Dismiss participants

Analysed $(n=20)$

- Excluded from analysis $(\mathrm{n}=0)$

\begin{tabular}{c}
\hline $\begin{array}{c}\text { Assessed for eligibility } \\
(\mathrm{n}=107)\end{array}$ \\
\hline
\end{tabular}

\section{Enrollment}

Excluded $(\mathrm{n}=67)$

- Not meeting inclusion criteria $(n=56)$

- Declined to participate $(n=7)$

- Other reasons $(n=4)$

\section{Experimental Group B $(\mathbf{n}=\mathbf{2 0})$}

- Review Informed Consent

- Confirm appropriate hygiene Cessation

- Brush teeth with control toothpaste

- Take intraoral photo

- $\quad$ Silness \& Loe Plaque Index

- Dismiss participants
Follow Up

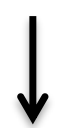

Analysis
Analysed $(\mathrm{n}=20)$

- Excluded from analysis $(\mathrm{n}=0)$

Figure 1. Experimental Design

assignment to a dental hygienist not involved in the study.

\section{Study design}

At baseline, participants were instructed to refrain from routine oral hygiene practices for 24 hours before their visit to our oral health centre. Upon their arrival, modified bass technique of brushing [18] was demonstrated to all of them. Both the groups were provided with a standardized toothbrush (Colgate ${ }^{\circledR}$ $360^{\circ}{ }^{\circledR}$ Advanced Optic White ${ }^{\circledR}$

Manual Toothbrush 360) and a similar amount of regular fluoridated toothpaste (Colgate Total ${ }^{\circledR}$ Clean Mint Toothpaste) was dispensed. All 


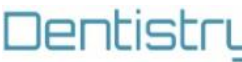

Vol 9 No 1 (2021) DOI 10.5195/d3000.2021.117

participants were asked to brush their teeth in front of a mirror to eliminate any compounding factors.

Immediately after brushing, a disclosing agent (GC Tri Plaque ID Gel $\left.{ }^{T M}\right)$ was applied to all tooth surfaces of all the participants with a micro brush to disclose the remaining plaque left after toothbrushing in both groups. The area was gently rinsed, and a cheek retractor was placed. Intraoral frontal photographs were taken by a DSLR camera with a macro lens for the analysis of the DPIA (Fig.2). Photographs were taken following the standard frontal photography requirements.

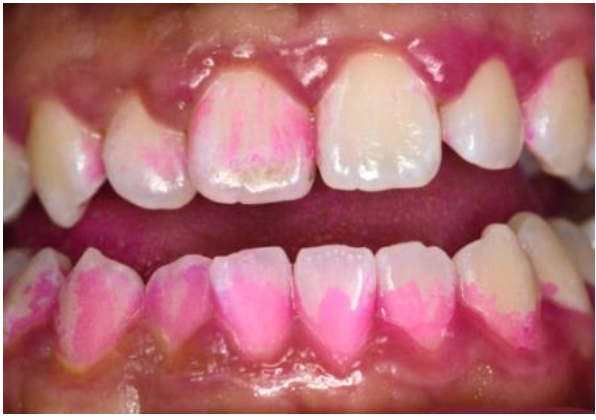

Figure 2. Intraoral frontal photographs taken for DPI analysis.

Next, biofilm left after toothbrushing was recorded using the Silness \& Loe Plaque Index (PI) [19]. Each of the four surfaces of the teeth (buccal, lingual, mesial and distal) were given a score from 0-3, where score 0 was absence of plaque, score 1 was a film of plaque adhering to the free gingival margin, score 2 was moderate plaque accumulation in sulcus or pocket and score 3 was abundance of soft matter in sulcus or pocket. The values obtained from this visit were recorded as baseline values for the clinical trial. Participants were then allowed to brush off the disclosing agent, and recalled after one month. Participants in both the groups were instructed not to use any other oral hygiene aids other than their toothbrush during this period.

Two trained examiners were calibrated in recording biofilm, prior to the commencement of the study, to ensure inter recorder reliability. The clinical parameters of $10 \%$ of the volunteers were evaluated in duplicate to obtain diagnostic confidence using the Kappa agreement test, whose inter-rater agreement was $k=0.89$. The examiners were blinded to the group allocation.

The second visit was planned one month after the first visit. Prior to the second visit, all participants were requested to refrain from brushing for 24 hours. On arrival at the oral health centre, the control group brushed with the regular fluoridated toothpaste (Colgate Total ${ }^{\circledR}$ Clean Mint Toothpaste) and the experimental group brushed with the experimental toothpaste (Shoplaq ${ }^{\circledR}$ disclosing toothpaste). Both the toothpastes contained fluoride, and were provided by the investigator. The experimental group was instructed to use one drop of dye containing toothpaste on a dry brush, and then to brush all the teeth and rinse, check teeth and gums for pink stains, and gently brush stains off. The brushing, disclosing, photographic procedures and plaque score recording were similar to the baseline visit.

Additionally, the experimental group was given a self-administered questionnaire at the end of the follow-up visit, to provide feedback towards the toothpaste used and side-effects if any.

The primary outcome was the amount of supragingival dental biofilm between the groups at the end of study and the secondary outcome was any side effects in the experimental group.

\section{Digital Image Analysis of Dental biofilm}

In addition to the clinical plaque scores, frontal photographs of the anterior teeth of subjects in both groups were taken after brushing and were subjected to DPIA analysis.

Frontal photographs were analyzed using computer software (MATLAB 2016 version). The photographs underwent a rough standardization of raw image size and were masked by a blinded investigator to define the area of analysis. RGB Standard Deviation detected the stains on the photographs. A pixel was deemed to be stained if the standard deviation (SD) of the RGB value exceeded a set percentage of the maximum SD observed in the image. 


\section{Dentistr}

Vol 9 No 1 (2021) DOI 10.5195/d3000.2021.117

A pixel at position $\mathrm{x}, \mathrm{y}$ was deemed stained if the following conditions were met:

$\frac{S D_{x, y}\left(R_{x, y}, G_{x, y}, B_{x, y}\right)}{\max \left\{S D_{x, y}\left(R_{x, y}, G_{x, y}, B_{x, y}\right)\right\}} \geq \sigma$

where $\max \left[S D_{x, y}\left(R_{x}, y, G_{x, y}, B_{x}, y\right)\right]$ was the maximal $S D$ value for all pixels in the processed image and $\sigma$ (ranging from 0 to 1) was a user-set sensitivity.

For image presentation, all stained pixels were then overlaid with a transparent cyan mask for ease of comparison. (Fig.3a\&3b) A simple relative area calculation was performed to quantify plaque coverage, as below:

$\%$ Plaque Coverage $=\frac{\text { no of pixels with stain }}{\text { total teeth pixels }} \times 100 \%$

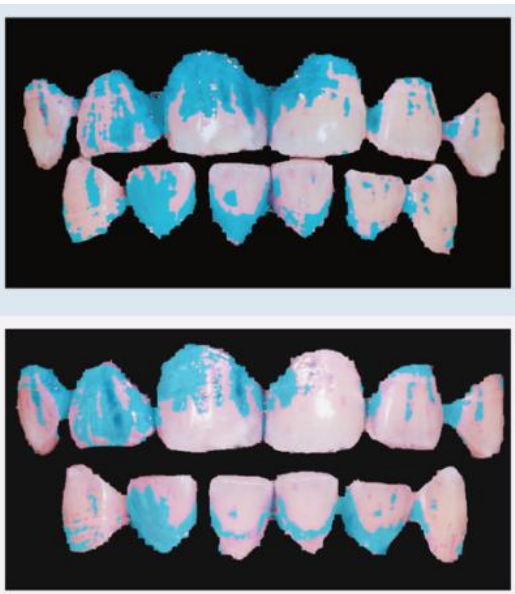

Figure $3 a$ \& $3 b$. Stained pixels overlaid with a transparent cyan mask to calculate percentage of plaque coverage after baseline and follow up visits respectively.

\section{Statistical Analysis}

Data was analyzed using SPSS version 22 (IBM Corporation, Armonk, NY, USA). Independent sample t-test was used to compare the mean plaque scores and mean DPIA scores between groups. $p<0.05$ was considered significant. Visual Analogue Scale (VAS) scores were expressed as a percentage.

\section{Results}

One hundred and seven patients were initially screened for eligibility. Of these, sixty-seven ( $n=67$ ) patients were excluded due to missing anterior teeth, ongoing orthodontic treatment, refusal to participate or if they did not meet the inclusion criteria. The remaining 40 subjects who were enrolled, completed the study, without any drop outs during the study period.

The mean age of the participants was $22.2 \pm 0.6$ years in control group and $21.8 \pm 0.3$ years in experimental group

Table 1. Comparison of Mean Plaque Scores between Control and Experimental Groups

\begin{tabular}{cccccc}
\hline Visit & Group & N & Mean & $\begin{array}{r}\text { Standard } \\
\text { Deviation }\end{array}$ & p-value \\
\hline \multirow{2}{*}{ Baseline } & Control & 20 & 1.16 & 0.49 & \\
\cline { 2 - 4 } & Experimental & 20 & 1.14 & 0.29 & 0.882 \\
\hline \multirow{2}{*}{ Follow up } & Experimental & 20 & 0.98 & 0.41 & 0.059
\end{tabular}

respectively. About $40 \%$ were males and $60 \%$ were females in both the groups. No differences were seen in the baseline plaque score of the groups with a mean plaque score of about 1.15 in both the groups. However, the follow up results showed that the mean plaque score was lesser $(0.98 \pm 0.41)$ in the group that used the dye containing toothpaste as compared to the other group (1.20 \pm 0.57$)$. But this difference was not significant with $\mathrm{p}=0.059$.

(Table 1)

Similarly, no significant differences were seen in the mean anterior plaque scores calculated using digital image analysis, either at the baseline or at the follow up visit with $p=0.98$ and 0.93 respectively. (Table 2 )

The questionnaire responses are shown in Table 3 and Table 4. About $65 \%$ of the participants in experimental group liked the color, freshness and cleansing action of the 
Vol 9 No 1 (2021) DOI 10.5195/d3000.2021.117

Table 2. Comparison of Mean DPIA scores between Control and Experimental Groups.

\begin{tabular}{cccccc}
\hline Visit & Group & N & Mean & $\begin{array}{c}\text { Standard } \\
\text { Deviation }\end{array}$ & p-value \\
& Control & 20 & 11.40 & 3.61 & \\
\multirow{2}{*}{ Baseline } & Experimental & 20 & 11.29 & 4.01 & 0.98 \\
\cline { 2 - 5 } & Control & 20 & 10.53 & 2.91 & \\
\hline \multirow{2}{*}{$\begin{array}{c}\text { Follow } \\
\text { up }\end{array}$} & Experimental & 20 & 10.25 & 2.87 & 0.93 \\
\hline
\end{tabular}

dye containing toothpaste and about

generally left behind after brushing

$70 \%$ felt motivated to brush using the

[24]. A simple means to improve

dye containing toothpaste. However,

about $40 \%$ felt that it took a longer

time to brush with the dye containing

toothpaste.

\section{Discussion}

The pathogenic nature of dental biofilm can be counteracted by maintaining effective oral hygiene, using mechanical and chemical approaches [20-22]. Home oral hygiene maintenance has shown to be ineffective in adults [23]. On average, $60 \%$ of the biofilm is one's oral hygiene habits is by improving skills involved in removal of dental biofilm [25].

A disclosing agent as the name itself suggests, changes the color of dental biofilm due to the dye present in it, which reveals the locations more clearly. This is due to the ability of dental biofilm to retain a large number of dye substances due to the interaction between the polarity difference of biofilm and dyes. However, the availability of these disclosing dyes in toothpaste is rare outside the dental offices [26]. Hence, in our study, the toothpaste Shoplaq ${ }^{\circ}$ was provided to the participants. The dye, $\mathrm{Cl}-45410$, present in this toothpaste gave it a pinkish color that adhered to the intraoral biofilm. The other ingredients included fluoride, natural herbs with no sodium lauryl sulphate added and it was a nonabrasive toothpaste.

In our study, no significant difference was observed in the overall mean plaque scores between the groups, which was similar to a study which also reported that disclosing agents did not play a critical role in improving plaque control [27]. However, studies by Stevens et al [10], and Ower et al [28] showed better plaque removal with the dye containing toothpaste. This could be attributed to the differences in the dye containing tooth pastes used, oral hygiene instructions provided prior to brushing and the indices used to record biofilm.

We felt that most areas such as the posterior teeth and lingual surfaces of the maxillary teeth which are often missed, cannot be seen while

Table 3: Experimental group's feedback on dye containing toothpaste $(n=20)$.

$\begin{array}{cccccc}\text { Items assessed } & \text { Very good } & \text { Good } & \text { Not sure } & \text { Bad } & \text { Very bad } \\ \text { Cleansing action } & 4(20 \%) & 9(45 \%) & 6(30 \%) & 1(5 \%) & 0 \\ \text { Colour } & 1(5 \%) & 11(55 \%) & 4(20 \%) & 3(15 \%) & 1(5 \%) \\ \text { Freshness } & 1(5 \%) & 13(65 \%) & 5(25 \%) & 1(5 \%) & 0 \\ \text { Time taken to brush } & 1(5 \%) & 6(30 \%) & 5(25 \%) & 5(25 \%) & 3(15 \%)\end{array}$


brushing. And not many people brush their teeth visualizing all areas in the mirror. Hence, the pink stains of the disclosed plaque could go unnoticed, even when a dye containing tooth paste was used. Hence, in our study, the biofilm on the anterior teeth was assessed using DPIA, anticipating that patients may visualize their anterior teeth better while brushing. However, no significant differences were seen in mean plaque scores between both groups using digital images. This indicates that correct technique of tooth brushing by patients is more important than merely using a disclosing dye containing toothpaste, for effective plaque removal.

Table 4: Experimental group's feedback on dye containing tooth paste.

\section{Items assessed Responses $\mathrm{n}=\mathbf{2 0}(\%)$}

\begin{tabular}{ll}
\hline \multicolumn{2}{l}{ Side effects } \\
\hline Yes & 0 \\
\hline No & $20(100 \%)$ \\
\hline \multicolumn{2}{l}{ Motivation to brush } \\
\hline Yes & $14(70 \%)$ \\
\hline No & $6(30 \%)$ \\
\hline Overall satisfaction & \\
\hline Satisfied & $7(35 \%)$ \\
\hline Neutral & $12(60 \%)$ \\
\hline Unsatisfied & $1(5 \%)$ \\
\hline
\end{tabular}

Establishing an accurate, visual method of assessing biofilm remaining after brushing is especially advantageous for children, elderly and patients undergoing orthodontic treatment for good oral hygiene. Adding a disclosing agent into the toothpaste, makes it more convenient for chairside education and is easily obtainable for the patient to bring home and can cultivate an effective brushing habit [29]. Our questionnaire responses showed that $70 \%$ of participants who used disclosing tooth paste, felt more motivated to brush their teeth. Hence, we can infer that disclosing toothpastes can still be a useful tool for educational and motivational purposes.

The limitations of this study were that the brushing technique was demonstrated only once and the time taken for brushing was not standardized. Also, the effectiveness of plaque removal was assessed after just one brushing, as our objective was solely to compare the effectiveness of the plaque disclosing toothpaste and not the effectiveness of patient's brushing technique. Hence, long term studies and different patient population pools are needed to verify the effectiveness of such products in future.

\section{Conclusion}

A dye containing toothpaste may not significantly improve plaque removal effectiveness as seen in our study; nevertheless, it may serve as a potential educational tool and motivate patients to maintain proper oral hygiene through a conscious effort.

\section{Acknowledgement}

The authors would like to thank the subjects for their patience and participation, Mr. Yeoh Chin Vern for his help in digital plaque analysis, $\mathrm{Dr}$ Khairiyah Abd Muttalib, Dr Bennete Fernandes and Dr Daniel Devaprakash for their critical evaluation and support throughout the study period.

\section{References}

1. Oral Microbes, Biofilms and Their Role in Periodontal and Peri-Implant Diseases. Lasserre JF, Brecx MC, Toma S. Materials (Basel). 2018 Sep 22;11(10):1802. doi: 10.3390/ma11101802. PMID: 30248991; PMCID: PMC6213094.

2. GBD 2016 Disease and Injury Incidence and Prevalence Collaborators. Global, regional, and national incidence, prevalence, and years lived with disability for 328 diseases and injuries for 195 countries, 19902016: a systematic analysis for the Global Burden of Disease Study 2016. Lancet. 2017 Sep 16; 390(10100):1211-1259. doi: 10.1016/S0140-6736(17)321542.

3. The antibacterial and antiplaque effectiveness of mouthwashes containing cetylpyridinium chloride with and without alcohol in improving gingival health. Williams MI. J Clin Dent. 
Vol 9 No 1 (2021) DOI 10.5195/d3000.2021.117

\section{1;22(6):179-82. PMID: 22403972}

4. Global oral health inequalities: task group--periodontal disease. Jin LJ, Armitage GC, Klinge B, Lang NP, Tonetti M, Williams RC. Adv Dent Res. 2011

May;23(2):221-6. doi: 10.1177/0022034511402080. PMID: 21490234.

5. Prevalence of periodontal disease, its association with systemic diseases and prevention. Nazir MA. Int J Health Sci (Qassim). 2017 AprJun;11(2):72-80. PMID: 28539867; PMCID: PMC5426403.

6. A global perspective on changes in the burden of caries and periodontitis: implications for dentistry. Baelum V, van Palenstein Helderman W, Hugoson A, Yee R, Fejerskov O. J Oral Rehabil. 2007 Dec;34(12):872-906; discussion 940. doi: 10.1111/j.13652842.2007.01799.x. PMID: 18034671.

7. A short-term clinical study design to investigate the chemical plaque inhibitory properties of mouthrinses when used as adjuncts to toothpastes: applied to chlorhexidine. Owens J, Addy M, Faulkner J, Lockwood C, Adair R J Clin Periodontol. 1997 Oct;24(10):732-7. doi: 10.1111/j.1600051x.1997.tb00190.x. PMID: 9350557.

8. Pannuti $\mathrm{CM}$, Saraiva MC, Ferraro A, Falsi D, Cai S, Lotufo RF. Efficacy of a $0.5 \%$ chlorhexidine gel on the control of gingivitis in Brazilian mentally handicapped patients. J Clin Periodontol. 2003 Jun;30(6):573-6. doi: 10.1034/j.1600051x.2003.00299.x. PMID: 12795797.
9. The importance of health education in prevention of oral health in children. Igić $M$, Apostolović M, Kostadinović L, Tricković-Janjić O, Surdilović D. Med Pregl. 2008 Jan-Feb;61(12):65-70. Serbian. doi: 10.2298/mpns0802065i. PMID: 18798477.

10. Evaluation of Plaque Removal Efficacy of a Novel DyeContaining Toothpaste: A Clinical Trial. Stevens K, Belavsky BZ, Evans CA, Viana G, Wu CD.Int J Dentistry Oral Sci. 03(1), 185 189.DOI : dx.doi.org/10.19070/2377-80751600039

11. Disclosing wafers in an oral hygiene instruction program. Bellini HT, Anerud A, Moustafa $\mathrm{MH}$. Odontol Revy. 1974;25(3):247-53. PMID: 4140484 .

12. The quantification of dental plaque using an image analysis system: reliability and validation. Smith RN, Brook AH, Elcock C. J Clin Periodontol. 2001

Dec;28(12):1158-62. doi: 10.1034/j.1600051x.2001.281211.x. PMID: 11737514.

13. Computerized planimetric method for clinical plaque measurement. Söder PO, Jin LJ, Söder B. Scand J Dent Res. 1993 Feb;101(1):21-5. doi: 10.1111/j.16000722.1993.tb01640.x. PMID: 8441891.

14. A study to assess the efficacy of a new detergent free, whitening dentifrice in vivo using QLF planimetric analysis. Pretty IA, Edgar WM, Higham SM.Br Dent J. 2004 Nov 13;197(9):561-6; discussion 551. doi: 10.1038/sj.bdj.4811809. PMID: 15543118
15. Refinement of the Modified Navy Plaque Index to increase plaque scoring efficiency in gumline and interproximal tooth areas. Rustogi KN, Curtis JP, Volpe AR, Kemp JH, McCool JJ, Korn LR. J Clin Dent. 1992;3(Suppl C):C9-12. PMID: 1306676.

16. New portable system for dental plaque measurement using a digital single-lens reflex camera and image analysis: Study of reliability and validation. Rosa GM, Elizondo ML. J Indian Soc Periodontol. 2015 MayJun;19(3):279-84. doi: 10.4103/0972-124X.152415. PMID: 26229267; PMCID: PMC4520111.

17. World Medical Association Declaration of Helsinki: ethical principles for medical research involving human subjects. World Medical Association. JAMA. 2013 Nov 27;310(20):2191-4. doi: 10.1001/jama.2013.281053. PMID: 24141714.

18. The Gingival Index, the Plaque Index and the Retention Index Systems. Löe H. J Periodontol. 1967 Nov-Dec;38(6):Suppl:6106. doi: 10.1902/jop.1967.38.6.610. PMID: 5237684.

19. Comparison of modified Bass technique with normal toothbrushing practices for efficacy in supragingival plaque removal. Poyato-Ferrera $\mathrm{M}$, Segura-Egea JJ, BullónFernández P. Int J Dent Hyg. 2003 May;1(2):110-4. doi: 10.1034/j.16015037.2003.00018.x. PMID: 16451532.

20. Self-reported changes in using fluoride toothpaste among older adults in Sweden: an intervention study. Acta Odontol Scand. Jensen O, Moberg Sköld 
Vol 9 No 1 (2021) DOI 10.5195/d3000.2021.117

U, Birkhed D, Gabre P. 2015

Jan;73(1):48-56. doi:

10.3109/00016357.2014.949847

. Epub 2014 Sep 3. PMID:

25183252.

21. Efficacy of two mouthwashes on 3-day supragingival plaque regrowth: a randomized crossover clinical trial. Marchetti

E, Casalena F, Capestro A, Tecco S, Mattei A, Marzo G Int J Dent Hyg. 2017 Feb;15(1):73-80. doi: 10.1111/idh.12185. Epub 2015 Nov 2. PMID: 26522915.

22. Oral hygiene measures and promotion: review and considerations. Choo A, Delac DM, Messer LB. Aust Dent J. 2001 Sep;46(3):166-73. doi: 10.1111/j.18347819.2001.tb00277.x. PMID: 11695154.

23. A systematic review of the effectiveness of self-performed mechanical plaque removal in adults with gingivitis using a manual toothbrush. van der Weijden GA, Hioe KP. J Clin Periodontol. 2005;32 Suppl 6:214-28. doi: 10.1111/j.1600051X.2005.00795.x. PMID: 16128840.

24. Plaque-left-behind after brushing: intra-oral reservoir for antibacterial toothpaste ingredients. Otten MP, Busscher $\mathrm{HJ}$, Abbas F, van der Mei HC, van Hoogmoed CG. Clin Oral Investig. 2012 Oct;16(5):143542. doi: 10.1007/s00784-0110648-2. Epub 2011 Dec 13. PMID: 22160537; PMCID: PMC3443356.

25. The effect of oral care feed-back devices on plaque removal and attitudes towards oral care.

Schäfer F, Nicholson JA, Gerritsen N, Wright RL, Gillam DG, Hall C. Int Dent J. 2003 Dec;53(6 Suppl 1):404-8. doi: 10.1111/j.1875- 595x.2003.tb00917.x. PMID: 14725386.

26. Mechanism of action of a twotone plaque disclosing agent. Gallagher IH, Fussell SJ, Cutress TW. J Periodontol. 1977 Jul;48(7):395-6. doi: 10.1902/jop.1977.48.7.395. PMID: 69704.

27. The role of visual feedback by a disclosing agent in plaque control. Tan AE, Wade AB. J Clin Periodontol. 1980 Apr;7(2):1408. doi: 10.1111/j.1600051x.1980.tb01957.x. PMID: 6155386.

28. The role of self-administered plaque control in the management of periodontal diseases: I. A review of the evidence. Ower P. Dent Update. 2003 Mar;30(2):60-4, 66, 68. doi: 10.12968/denu.2003.30.2.60. PMID: 12705026.

29. Detection of dental plaque with disclosing agents in the context of preventive oral hygiene training programs. Fasoulas $\mathrm{A}$, Pavlidou E, Petridis D, Mantzorou M, Seroglou K, Giaginis C. Heliyon. $2019 \mathrm{Jul}$ 10;5(7):e02064. doi: 10.1016/j.heliyon.2019.e02064. PMID: 31334380; PMCID: PMC6624240. 